and Infectious Diseases, Bethesda) and his colleagues at the international symposium on medical and applied virology in Fort Lauderdale on December 14-17, and at the Gustav Stern symposium on perspectives in virology at New York on February 2-3.

Baron et al. first examined the effect of poly-I : C on experimental rhinovirus infection in humans. When the dose estimated to confer protection, and still be safe, was given to volunteers, working up from one tenth of the dose, no evidence of toxicity was found. The subjects for trials against experimental virus infection were eighteen healthy volunteer prisoners, selected for a low concentration of serum neutralizing antibody to rhinovirus-13, so that they should be susceptible to infection. On the day before virus challenge, eight men were given $0 \cdot 1 \mathrm{mg} / \mathrm{kg}$ body weight of poly-I : C intranasally; the rest received placebos. Further doses of poly-I : $\mathrm{C}$ or placebo were given for 5 days after infection, and the men were examined independently for symptoms. Five out of ten of those given placebos developed colds, compared with one in eight of those who received poly-I : C. And fewer viruses were recovered from the nasal washings of treated men. Probably more significant than the development of colds was the overall reduction in symptoms in the treated group, as measured by symptom score analysis.

When influenza virus was the challenge there was again a reduction in disease and symptoms-illness occurred in four out of nine subjects treated with poly-I: $\mathrm{C}$ and in seven out of ten given placebos.

Baron et al. also investigated the relationship between interferon titre and doses of poly-I : $\mathrm{C}$ in patients with either encephalitis or cancer, to look for ways of preserving a high titre of interferon for the longer periods necessary in prophylactic treatment. After one dose of poly-I : $\mathrm{C}$ there was a decrease in interferon after 24 hours, reaching a minimum after about 3 days. Further doses given during this period of decline had no effect. But in some cancer patients, treated intravenously, a dose given 7 days after the initial dose induced some interferon. Intravenous injections caused fever, reversible suppression of erythrocyte formation, and in one patient an allergic reaction.

These results are all encouraging, although only a few subjects were involved, both for the protection afforded by poly-I : $\mathrm{C}$ and for the apparent lack of side effects from intranasal treatment. On the question of toxicity, it should be remembered that experimental virus challenge doses are usually much higher than those occurring in natural infection, and so much less interferon inducer should be necessary for prophylaxis, with a reduction in possible toxicity. Certainly this is an interesting beginning to the therapeutic use of a synthetic interferon inducer in humans.

\section{OXYGEN SENSORS Galvanic Cell makes Good}

from a Correspondent

Some useful new developments in the measurement of partial pressures of oxygen were described when members of the Analytical Group of the Society of Analytical Chemistry met in London on March 12 to discuss oxygen sensors in biological and other systems. There has been growing interest in the direct measurement of the partial pressure of oxygen, particularly for medical purposes and in closed environments such as space capsules and mines. The polarographic oxygen electrode of Clark has been most commonly used to measure oxygen partial pressure in fluids.

Dr I. Bergman (Safety in Mines Research Establishment, Sheffield) pointed out that the galvanic oxygen electrode, which unlike the Clark electrode requires no external applied voltage to bring about the reduction of oxygen at the cathode, is essentially a polarographic device when operated in the gas diffusion controlled region. Research at the Safety in Mines Research Establishment has led to the development of several polarographic oxygen sensors in which a silver cathode is evaporated on to a polytetrafluoroethylene (PTFE) membrane. The anode is $\mathrm{Ag} / \mathrm{AgCl}$. In this way very efficient reduction of the incoming oxygen is achieved as well as a very fast response time. Bergman demonstrated an oxygen sensor which could be used to control the partial pressure of oxygen in an oxygen tent used in hospital during the care of premature babies.

Dr D. Parker and Mr A. Key (University College Hospital, London) reported the development of three silver-lead galvanic cells for measuring oxygen tension in blood. The first was a "flow-through" sensor which measured continuously the oxygen tension of blood flowing from a heart-lung machine to a patient undergoing heart surgery. The sensor electrodes were isolated from the blood by a PTFE membrane. Temperature compensation was incorporated in the device so that the cell output was linear in the range $20^{\circ}-40^{\circ}$ $\mathrm{C}$ for a $p \mathrm{O}_{\mathrm{c}}$ from $0.710 \mathrm{~mm} \mathrm{Hg}$. The second sensor was a small probe used to determine glucose concentration in serum and urine. The glucose sample was added to an air-saturated glucose oxidase enzyme and the rate of consumption of oxygen measured by the oxygen sensor. The rate of consumption was proportional to the concentration of glucose initially present.

Key reported the development of an in vivo silverlead galvanic oxygen sensor incorporated into the tip of a PTFE catheter of $0.8 \mathrm{~mm}$ diameter. A preformed PTFE membrane fits over the catheter tip. The catheter oxygen sensor is guided into the heart where the measurement of oxygen can be used to detect shunts across the chambers of the heart. The device could become a valuable diagnostic tool for the early detection of diseases of the heart.

There is a great deal of interest in galvanic cells as in vivo energy sources in which oxygen from the body fluids is converted directly to electrical power. In this way it is hoped to provide in vivo the energy to power cardiac pacemakers. At present, batteries implanted in the human body have a useful life of 18-24 months and thus their replacement necessitates expensive surgery about every two years. Dr A. C. C. Tseung (City University, London) reported the use of a platinum black-aluminium galvanic cell as an in vivo energy source. The cell electrolyte was an ionic ion-exchange membrane and the cell is encapsulated in silicone rubber. Such a cell planted subcutaneously near the abdominal wall of a rat gave a power output during intermittent use of $100 \mu \mathrm{W}$ at $l \cdot 1 \mathrm{~V}$ for a total of $500 \mathrm{~h}$. This was well above the objective of $30 \mu \mathrm{W}$ at $1 \cdot 1 \mathrm{~V}$.

If the problem of the deposition of contaminants from the body on the cell membrane leading to a decrease in the membrane permeability for oxygen can. be overcome, the galvanic cell promises seriously to rival present in vivo energy sources. 